\title{
Data Volume Reduction in High-Resolution Wide-Swath SAR Systems
}

\author{
Michelangelo Villano, Gerhard Krieger, Alberto Moreira \\ Microwaves and Radar Institute \\ German Aerospace Center (DLR) \\ Wessling, Germany \\ michelangelo.villano@dlr.de
}

\begin{abstract}
High-resolution wide-swath (HRWS) synthetic aperture radar (SAR) systems are very attractive for the observation of dynamic processes on the Earth's surface, but they are also associated with a huge data volume. In order to comply with azimuth ambiguity requirements, in fact, a pulse repetition frequency (PRF) much higher than the required processed Doppler bandwidth (PBW) is often desirable. The data volume can be drastically reduced, if on-board Doppler filtering and decimation are performed prior to downlink. A finite impulse response (FIR) filter with a relatively small number of taps suffices to suppress the additional ambiguous components and recover the original impulse response. This strategy is also applicable and especially relevant to staggered SAR systems, where on-board Doppler filtering and resampling can be jointly implemented.
\end{abstract}

Index Terms-Synthetic aperture radar (SAR), high-resolution wide-swath (HRWS) imaging, staggered SAR, data volume reduction, finite impulse response (FIR) filter, on-board processing.

\section{INTRODUCTION}

Synthetic aperture radar (SAR) is a remote sensing technique, capable of providing high-resolution images independent of weather conditions and sunlight illumination. This makes SAR very attractive for the systematic observation of dynamic processes on the Earth's surface [1]. However, conventional SAR systems are limited, in that a wide swath can only be achieved at the expense of a degraded azimuth resolution. This limitation can be overcome by high-resolution wide-swath (HRWS) systems based on digital beamforming (DFB) on receive, where multiple swaths can be simultaneously imaged using multiple elevation beams [2]. Moreover, if the system is operated in staggered SAR mode, i.e., if the pulse repetition interval (PRI) is continuously varied, it is also possible to get rid of the "blind ranges", present between adjacent swaths, as the radar cannot receive while it is transmitting [3], [4].

Due to their resolution and coverage requirements, HRWS systems are inherently associated with a huge data volume, thereby increasing the demands for internal data storage, downlink, ground processing and archiving. Recent studies related to Tandem-L, a proposal for a polarimetric and interferometric satellite mission to monitor dynamic processes over the Earth's surface with unprecedented accuracy and resolution, quantify the volume of the acquired data as 8 TB/day [5].

Moreover, in order to comply with azimuth ambiguity requirements, a pulse repetition frequency (PRF) much higher than the required processed Doppler bandwidth (PBW) is often desirable. For a HRWS SAR system with constant PRI and multiple elevation beams, in order to achieve a good azimuth ambiguity-to-signal ratio (AASR), the required PRF is usually even larger than twice the PBW. As an example, in a SAR system with PRF $=1800 \mathrm{~Hz}$ and $\mathrm{PBW} B_{p}=780 \mathrm{~Hz}$, the data volume to be downlinked increases by more than $130 \%$ due to the azimuth oversampling. The system, in fact, downlinks data included in the Doppler frequency interval [-PRF/2, PRF/2], while only data in the Doppler frequency interval $\left[-B_{p} / 2, B_{p} / 2\right]$ are needed to achieve the desired azimuth resolution. The information contained in the Doppler frequency intervals [$\left.\mathrm{PRF} / 2,-B_{p} / 2\right]$ and $\left[-B_{p} / 2, \mathrm{PRF} / 2\right]$ is useless and discarded in the SAR processing.

If the system is operated in staggered SAR mode, the ratio of the mean PRF on transmit to the PBW can be even larger than 3. This determines a further increase of the data volume to be downlinked with a direct impact on the cost of the mission. As an example, for a staggered SAR system with a mean PRF on transmit $P R F_{\text {meanTX }}$ equal to $2700 \mathrm{~Hz}$ and a $\mathrm{PBW} B_{p}=780$ $\mathrm{Hz}$, the data volume to be downlinked increases by almost $250 \%$.

\section{DATA VOLUME REDUCTION CONCEPT}

Let us first consider the case of a SAR system with constant PRI. If data were just decimated prior to downlink (e.g., by a factor of 2 in the latter example where PRF $=1800 \mathrm{~Hz}$ and $B_{p}$ $=780 \mathrm{~Hz}$ ), a considerable degradation of the AASR would occur. Fig. 1 (a) shows the power spectral density (PSD) of the azimuth SAR signal at near range for an L-band reflector antenna with a diameter of $15 \mathrm{~m}$. The PSD is the joint transmitreceive antenna pattern, displayed as a function of Doppler frequency. The unambiguous energy, the ambiguous energy, and the additional ambiguous energy due to the decimation are highlighted in green, red, and blue, respectively. As is apparent, the additional ambiguous energy due to decimation is significant, i.e., the total ambiguous energy is the same obtained for PRF $=1800 \mathrm{~Hz} / 2=900 \mathrm{~Hz}$. However, if Doppler filtering is performed before decimation, the additional 
ambiguous energy due to decimation can be substantially reduced, as shown in Fig. 1 (b) [6] $]^{1}$.

Due to the large amount of data, acquired by typical HRWS systems, the number of on-board operations per sample has to be minimized, while avoiding a degradation of the impulse response. The Doppler filtering can be therefore performed in time domain using a finite impulse response (FIR) filter with a relatively small number of taps. The filter will introduce a distortion of the Doppler spectrum of the signal, which can be compensated for in the SAR processing (on ground). The case of decimation by an integer factor is analyzed in the following, as this is associated with a straightforward implementation and a much lower computational cost, but the proposed strategy can be also used in case of a rational decimation factor.

In a staggered SAR system, the Doppler filter has to be applied to raw data resampled to a uniform PRI, but, in practice, resampling, Doppler filtering, and decimation can be also jointly performed, as explained in the following. Fig. 2 shows the block diagrams of the proposed data volume reduction strategy for a system with constant PRI and a staggered SAR system.

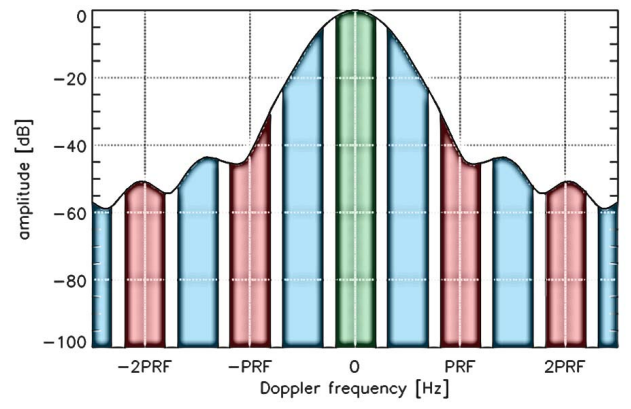

(a)

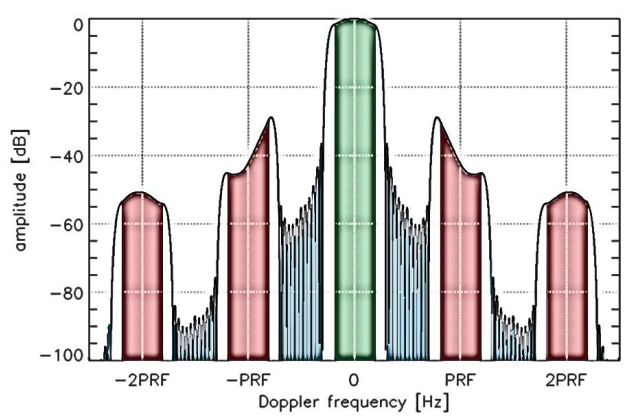

(b)

Fig. 1. PSD of the azimuth SAR signal at near range for an L-band reflector with $15 \mathrm{~m}$ diameter. The energy of the unambiguous component, the ambiguous components, and the additional ambiguous components due to decimation are highlighted in green, red and blue, respectively.

(a) Only decimation (no Doppler filtering). (b) Doppler filtering and decimation.

Fig. 3 (a) shows how in the staggered SAR case the filtering has to be applied on resampled data, which are obtained from the raw staggered SAR (non-uniformly sampled)

${ }^{1}$ The azimuth presumming, used so far in airborne SAR, can be considered as a Doppler filtering and represents the easiest way of data reduction. data through best linear unbiased (BLU) interpolation. Each sample of the resampled data is obtained as a linear combination of some of the samples of the raw staggered SAR data, while each sample of the filtered data is obtained as a linear combination of some of the resampled data. This means that each sample of the filtered data can be obtained directly as a linear combination of some of the staggered SAR data (Fig. 3 (b)). Moreover, there is no need to compute the samples which would anyway be discarded by the decimation operation.

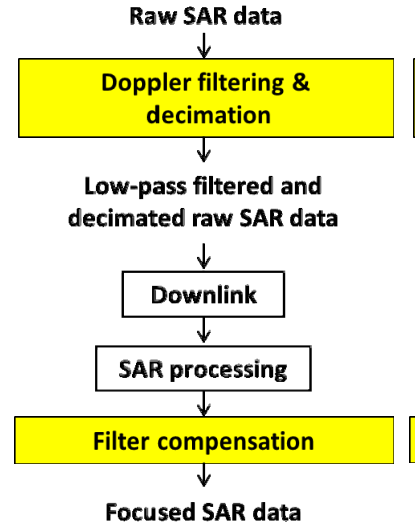

(a)

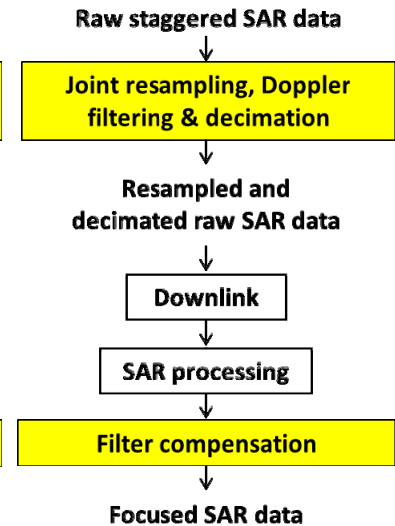

(b)
Fig. 2. Block diagram of the proposed data volume reduction strategy. (a) Constant PRI SAR. (b) Staggered SAR.

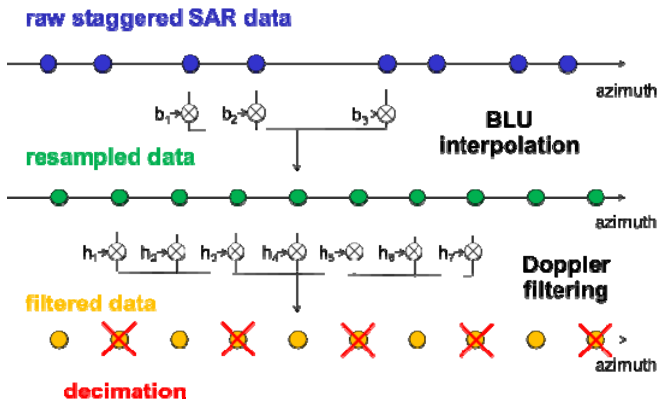

(a)

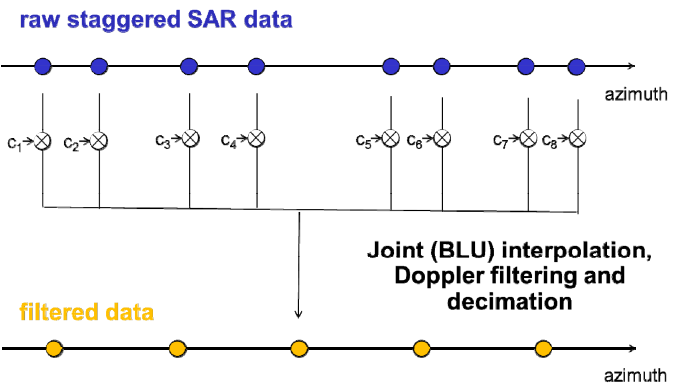

(b)

Fig. 3. (a) Interpolation, Doppler filtering and decimation in the staggered SAR case. (b) Equivalent scheme, where interpolation, Doppler filtering and decimation are jointly performed. 


\section{FILTER DESIGN}

The FIR filter can be designed as a Wiener filter, i.e., exploiting the knowledge of the PSD of the useful and disturbance signals [7]. In this case, the disturbance signal is given by the frequency components, which fold back to the main part of the spectrum after decimation. The coefficients of the $P$-tap FIR Wiener filter are given by

$$
\mathbf{h}=\mathbf{R}_{\mathbf{s}}^{-1} \mathbf{r}_{\mathbf{u}}
$$

where $\mathbf{R}_{\mathbf{s}}$ is the correlation matrix of the overall signal and $\mathbf{r}_{\mathbf{u}}$ is the correlation vector of the useful signal, given by

$$
\mathbf{R}_{\mathbf{s}}=\left[\begin{array}{cccc}
R_{s}[0] & R_{s}[-1] & \cdots & R_{s}[1-P] \\
R_{s}[1] & R_{s}[0] & \cdots & R_{s}[2-P] \\
\vdots & \vdots & \ddots & \vdots \\
R_{s}[P-1] & R_{s}[P-2] & \cdots & R_{s}[0]
\end{array}\right]
$$

and

$$
\mathbf{r}_{\mathbf{u}}=\left[\begin{array}{c}
R_{u}\left[-\frac{P-1}{2}\right] \\
\vdots \\
R_{u}[0] \\
\vdots \\
R_{u}\left[\frac{P-1}{2}\right]
\end{array}\right]
$$

respectively. For a decimation factor equal to $2, R_{s}[n]$ and $R_{u}[n]$ are related to the two way power pattern in azimuth $G^{2}(f)$ through the following relation

$$
\begin{aligned}
R_{s}[n]= & R_{u}[n]+R_{d}[n] \\
& =\frac{2}{P R F} \int_{0}^{B_{p} / 2} G^{2}(f) \cos \left(\frac{2 \pi n}{P R F} f\right) d f \\
& +\frac{2}{P R F} \int_{\left(P R F-B_{p}\right) / 2}^{P R F / 2} G^{2}(f) \cos \left(\frac{2 \pi n}{P R F} f\right) d f
\end{aligned}
$$

where a symmetric antenna azimuth pattern has been assumed and all back-folded frequency components of second and higher order have been ignored.

Fig. 4 (a) shows the transfer function of the 25-tap FIR Wiener filter obtained for $P R F=1800 \mathrm{~Hz}$ and $B_{p}=780 \mathrm{~Hz}$, assuming the azimuth pattern at near range of the aforementioned L-band reflector antenna and a decimation of the data by a factor of 2 . It can be noticed how the filter attenuates the frequency components in the Doppler frequency interval $\left[\mathrm{PRF} / 2-B_{p} / 2, \mathrm{PRF} / 2\right]$. For this decimation factor, in fact, a low-pass filter is needed.

An alternative to design the FIR Wiener filter is given by the minimum variance distortionless response (MVDR) or Capon beamformer [8], where only the knowledge of the PSD of the disturbance signal is exploited. The coefficients of the MVDR Wiener filter are given by

$$
\mathbf{h}=\mathbf{R}_{\mathbf{d}}^{-\mathbf{1}} \mathbf{1}
$$

where $\mathbf{R}_{\mathbf{d}}$ is the correlation matrix of the disturbance signal, given by

$$
\mathbf{R}_{\mathbf{d}}=\left[\begin{array}{cccc}
R_{d}[0] & R_{d}[-1] & \cdots & R_{d}[1-P] \\
R_{d}[1] & R_{d}[0] & \cdots & R_{d}[2-P] \\
\vdots & \vdots & \ddots & \vdots \\
R_{d}[P-1] & R_{d}[P-2] & \cdots & R_{d}[0]
\end{array}\right]
$$

and $\mathbf{1}$ is a steering vector, whose components are all ones. The relationship between $R_{d}[n]$ and the two way power pattern $G^{2}(f)$ is given for a decimation factor equal to 2 in (4). Fig. 4 (b) shows the filter coefficients and the transfer function of the 9-tap MVDR filter obtained for the same antenna pattern and parameters.

While the Wiener filter is characterized by a flat response in the Doppler frequency interval $\left[0, B_{p} / 2\right]$ and requires more taps (i.e., 25 in the example of Fig. 4 (a)) to provide a sufficient attenuation in the interval $\left[\mathrm{PRF} / 2-B_{p} / 2, \mathrm{PRF} / 2\right]$, the MVDR achieves a very good suppression of the higher frequencies with a much smaller number of taps (i.e., 9 in the example of Fig. 4 (b)). As a drawback, the MVDR filter introduces a significant attenuation in the interval $\left[0, B_{p} / 2\right]$ as well (up to 20 $\mathrm{dB}$ in the example of Fig. 4 (b)), which can be however compensated in the processing, as explained in the following.

For a staggered SAR system the same formulas for the design of the filter hold, where in place of the PRF, the uniform PRF to which non-uniformly sampled data are resampled has to be used. The latter PRF can be selected equal to the mean PRF on transmit of the system, as done in the following example, but it can also be different, therefore allowing in a straightforward way to obtain a decimation by an arbitrary noninteger decimation factor. With reference to an L-band staggered SAR system, where the mean PRF on transmit is equal to $P R F_{\text {mean } T X}=2700 \mathrm{~Hz}$ and the $\mathrm{PBW}$ is $B_{p}=780 \mathrm{~Hz}$, data could be resampled to a uniform $\mathrm{PRF}=P R F_{\text {mean }} T X$, filtered, and finally decimated by a factor of 3 to $900 \mathrm{~Hz}$.

For a decimation factor equal to $3, R_{s}[n]$ and $R_{u}[n]$ are related to the two-way power pattern in azimuth $G^{2}(f)$ through the following relation

$$
\begin{aligned}
R_{s}[n]= & R_{u}[n]+R_{d}[n] \\
& =\frac{2}{P R F} \int_{0}^{B_{p} / 2} G^{2}(f) \cos \left(\frac{2 \pi n}{P R F} f\right) d f \\
& +\frac{2}{P R F} \int_{P R F / 3-B_{p} / 2}^{P R F / 3+B_{p} / 2} G^{2}(f) \cos \left(\frac{2 \pi n}{P R F} f\right) d f
\end{aligned}
$$

always assuming a symmetric antenna azimuth pattern centered about zero Doppler and ignoring back-folded high-frequency components. Fig. 4 (c) shows the filter coefficients and the transfer function so obtained. As apparent, the filter for data volume reduction for a decimation factor equal to 3 is no longer a low-pass filter, but instead a band-stop filter. 


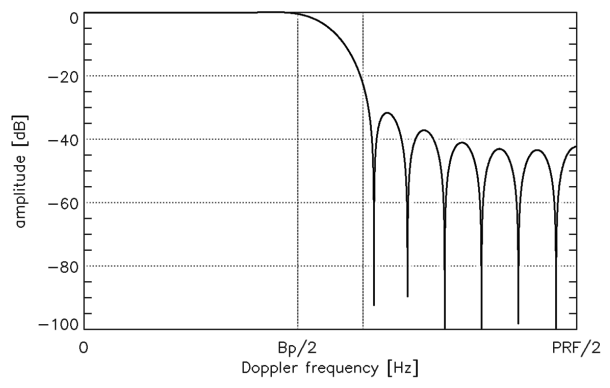

(a)

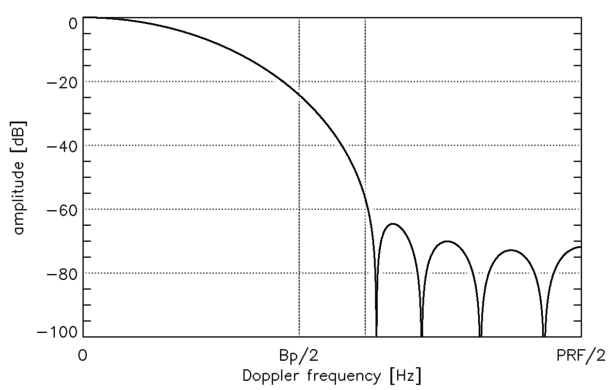

(b)

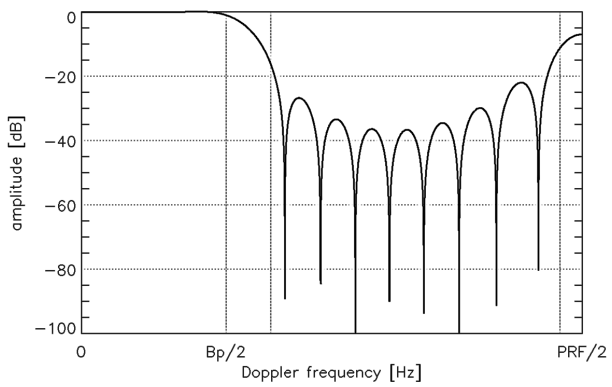

(c)

Fig. 4. Transfer functions of the designed data volume reduction filters.

(a) 25-tap Wiener filter used for data volume reduction by a factor of 2 (constant PRI SAR). (b) 9-tap MVDR filter used for data volume reduction by a factor of 2 (constant PRI SAR). (c) 25-tap Wiener filter used for data volume reduction by a factor of 3 (staggered SAR).

\section{PERFORMANCE ANALYSIS}

The performance of a system, where the described data volume reduction strategy is implemented, is evaluated and compared with a reference system, where the data volume reduction strategy is not applied, i.e., all data are downlinked. Some considerations of the joint effect of Doppler filtering and quantization are also reported.

The azimuth resolution and the azimuth peak-to-sidelobe ratio (PSLR) remain unchanged with respect to the reference case, provided that the distortion of the Doppler spectrum of the signal, introduced by the low-pass filtering, is compensated for in the processing. This is done by multiplying the azimuth spectrum of the processed data by $C(f)$, where

$$
C(f)= \begin{cases}\frac{1}{H(f)} & ,|f| \leq B_{p} / 2 \\ 0 & , B_{p} / 2 \leq|f| \leq P R F / 2\end{cases}
$$

where $H(f)$ is the transfer function of the employed FIR filter. As far as azimuth ambiguities are concerned, the AASR for a SAR system with constant PRI, where the described data volume reduction strategy is applied, for an integer decimation factor $p$ and assuming that $\mathrm{PRF} \geq p B_{p}$, can be analytically expressed as

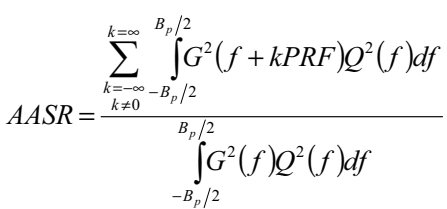

$$
+\frac{\sum_{k=-\infty}^{k=\infty} \sum_{h=1}^{p-1} \int_{-B_{p} / 2}^{B_{p} / 2} G^{2}\left(f+\left(k+\frac{h}{p}\right) P R F\right) Q^{2}(f) \frac{H^{2}\left(f+\frac{h}{p} P R F\right)}{H^{2}(f)} d f}{\int_{-B_{p} / 2}^{B_{p} / 2} G^{2}(f) Q^{2}(f) d f}
$$

where $Q(f)$ accounts for the amplitude weighting of the Doppler spectrum applied in the processing (e.g., Hamming window and compensation of the azimuth antenna pattern), not including the compensation of the low-pass filter. The AASR is composed of two terms, where the first term is the AASR of a system, where no data volume reduction is performed, while the second one represents the AASR degradation due to the onboard filtering.

Fig. 5 (a) shows the AASR as a function of ground range for a SAR with constant PRI and multiple elevation beams, an L-band reflector antenna with $15 \mathrm{~m}$ diameter, $\mathrm{PRF}=1800 \mathrm{~Hz}$, and $B_{p}=780 \mathrm{~Hz}$, assuming that data volume reduction is not performed. The AASRs for the case where the data are decimated by a factor of 2, using both the Wiener filter of Fig. 4 (a) and the MVDR filter of Fig. 4 (b), are superimposed. As apparent, the proposed strategy based on Doppler filtering and decimation allows a significant reduction of the data volume at the expense of a negligible AASR degradation. The AASR degradation, defined as the difference of the AASRs obtained with and without data volume reduction, i.e., defined as the second term of (9), is displayed in Fig. 5 (b). This is smaller than $-48 \mathrm{~dB}$ for the 25-tap Wiener filter and smaller than -62 $\mathrm{dB}$ for the 9-tap MVDR filter.

This AASR degradation is reflected in the 2D impulse response through additional azimuth ambiguities, which, in case of decimation by a factor of 2 , are located at half the azimuth distance of the first-order azimuth ambiguities, as can be observed in Fig. 6, where the 2D impulse responses at near range are shown in case data volume reduction is not performed (Fig. 6 (a)) and in case the 25-tap Wiener filter of Fig. 4 (a) is used (Fig. 6 (b)). The additional azimuth ambiguities are due to decimation, while the Doppler filter significantly reduces their energy. 


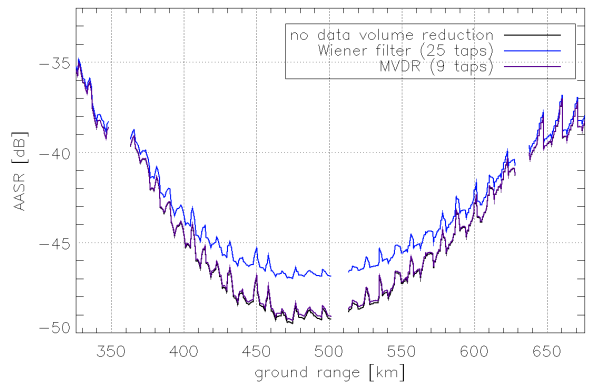

(a)

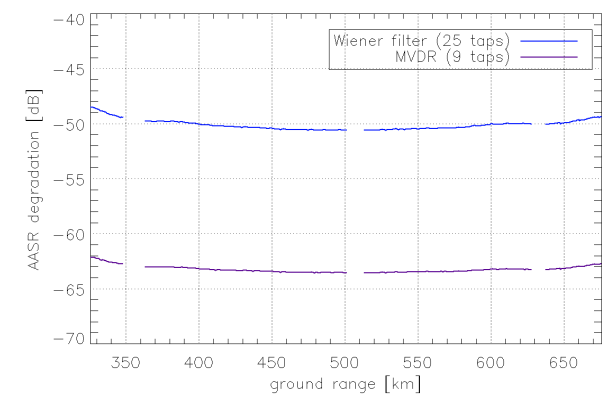

(b)

Fig. 5. (a) AASR vs. ground range for a constant PRI SAR in case data volume reduction is not performed and in case data volume reduction by a factor of 2 is performed using the Wiener filter of Fig. 4 (a) and the MVDR filter of Fig. 4 (b). The black and the purple curves almost overlap. (b) AASR degradation due to data volume reduction.

In the staggered SAR case the AASR can be evaluated by simulation as the difference of ISLRs [9]. Fig. 7 shows the AASR as a function of ground range for a staggered SAR with $\mathrm{PRF}_{\text {mean } \mathrm{TX}}=2700 \mathrm{~Hz}$, and $\mathrm{B}_{\mathrm{p}}=780 \mathrm{~Hz}$, assuming that data volume reduction is not performed. The AASR in case data are decimated by a factor of 3, using the Wiener filter of Fig. 4 (c), is superimposed. As apparent, in the staggered SAR case the proposed strategy based on Doppler filtering and decimation allows an even more significant reduction of the data volume at the expense of a negligible AASR degradation, not even visible in the AASR plot. It has therefore to be considered as an integral part of the staggered SAR concept.

This AASR degradation is reflected in the 2D impulse response even in the staggered SAR case through localized additional azimuth ambiguities, as it can be observed in Fig. 8, where the $2 \mathrm{D}$ impulse responses at near range are shown in case data volume reduction is not performed (Fig. 8 (a)) and in case the 25-tap Wiener filter of Fig. 4 (c) is used (Fig. 8 (b)).

In the context of data volume reduction, the on-board Doppler filtering is likely to be followed by a quantization stage prior to downlink. The joint effects of Doppler filtering and quantization have been addressed in [10], where SAR raw data acquired by the German satellite TerraSAR-X over the Amazon rainforest have been used for the analysis. The outcome of the analysis is that a degradation of the noise equivalent sigma zero (NESZ) occurs for the MVDR filter, which can be accepted, if the on-board computational capacity only allows using a filter with a smaller number of taps.
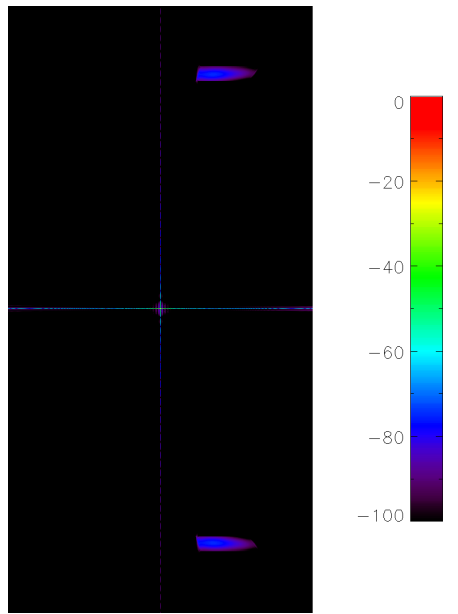

(a)
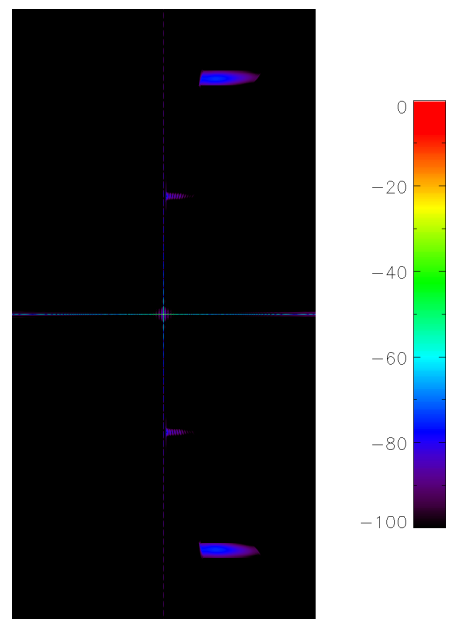

(b)

Fig. 6. Effect of data volume reduction on the $2 \mathrm{D}$ IRF for a constant $P R I$ SAR. The horizontal and vertical axes represent slant range and azimuth, respectively. The size (slant range $\times$ azimuth) is $0.9 \mathrm{~km} \times 20.3 \mathrm{~km}$. (a) $2 \mathrm{D}$ IRF in $\mathrm{dB}$, in case no data volume reduction is performed. (b) $2 \mathrm{D}$

IRF in $\mathrm{dB}$, in case data are decimated by a factor of 2 , after having filtered them with the 25-tap Wiener filter of Fig. 4 (a). The additional azimuth ambiguities due to decimation are visible, but negligible (AASR degradation smaller than $-48 \mathrm{~dB}$ ).

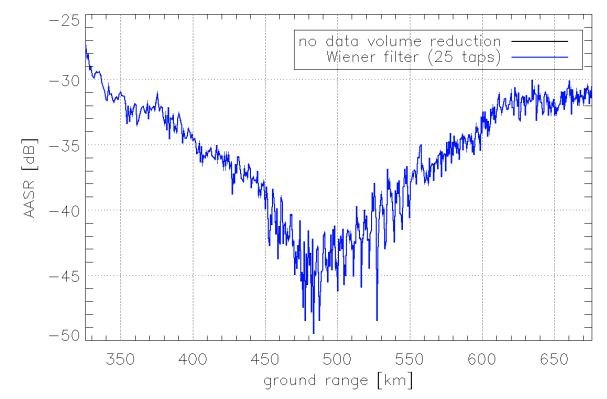

Fig. 7. $A A S R$ vs. ground range for a staggered SAR in case data volume reduction is not performed and in case data volume reduction by a factor of 3 is performed using the Wiener filter of Fig. 4 (c). The two curves almost overlap. 

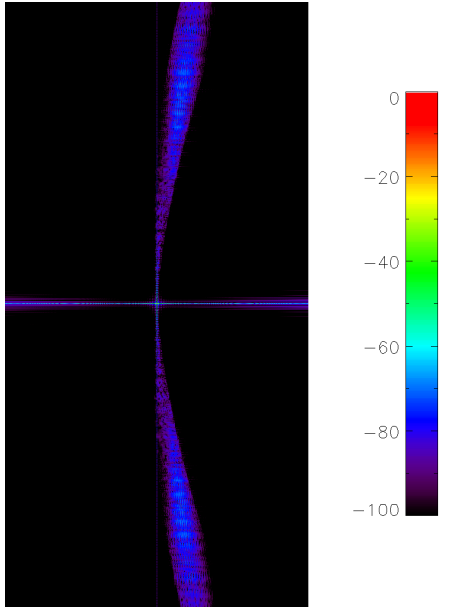

(a)
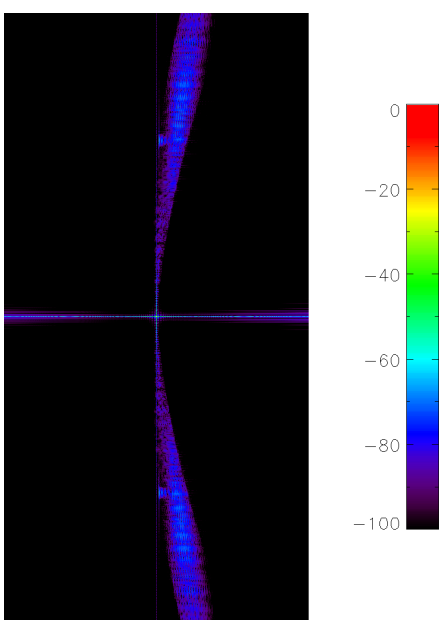

(b)

\section{REFERENCES}

[1] A. Moreira et al., "A Tutorial on Synthetic Aperture Radar," IEEE Geoscience and Remote Sensing Magazine, vol. 1, no. 1, 2013.

[2] G. Krieger et al., "Advanced concepts for ultra-wide-swath SAR imaging," Proceedings of EUSAR, Friedrichshafen, Germany, 2008.

[3] M. Villano, G. Krieger, A. Moreira, "Staggered SAR: HighResolution Wide-Swath Imaging by Continuous PRI Variation," IEEE Trans. Geosci. Remote Sens., vol. 52, No. 7, pp. 44624479, 2014.

[4] M. Villano, G. Krieger, A. Moreira, "A Novel Processing Strategy for Staggered SAR," IEEE Geosci. Remote Sens.Lett., vol. 11, No. 11, pp. 1891-1895, 2014.

[5] A. Moreira et al., "Tandem-L: A Highly Innovative SAR Mission for Global Observation of Dynamic Processes on the Earth's Surface," IEEE Geoscience and Remote Sensing Magazine, vol. 3, no. 2, 2015, in press.

[6] M. Villano, G. Krieger, and V. Del Zoppo, "On-Board Doppler Filtering for Data Volume Reduction in Spaceborne SAR Systems," Proceedings of the IRS, Gdansk, Poland, 2014.

[7] S. M. Kay, "Fundamentals of Statistical Signal Processing, vol. 1, Upper Saddle River," NJ, USA: Prentice-Hall, 1993.

[8] J. Capon, "High resolution frequency-wavenumber spectrum analysis," Proc. IEEE, vol. 57, pp. 1408-1418, Aug. 1969.

[9] M. Villano, G. Krieger, A. Moreira, "Ambiguities and Image Quality in Staggered SAR," Proceedings of the APSAR Conference, Marina Bay Sands, Singapore, 2015, in press.

[10] M. Villano, M. Martone, V. Del Zoppo, and G. Krieger, "Joint effects of on-board Doppler filtering and quantization in spaceborne SAR systems," Proceedings of the IEEE GOLD Remote Sensing Conference, Berlin, Germany, 2014.

Fig. 8. Effect of data volume reduction on the $2 \mathrm{D}$ IRF for a staggered SAR. The horizontal and vertical axes represent slant range and azimuth, respectively. The size (slant range $\times$ azimuth) is $1.7 \mathrm{~km} \times 40.6 \mathrm{~km}$. (a) 2D IRF in $\mathrm{dB}$, in case all data are downlinked. (b) $2 \mathrm{D}$ IRF in $\mathrm{dB}$, in case data are decimated by a factor of 3 , after having filtered them with the 25-tap Wiener filter of Fig. 4 (c). The additional localized azimuth ambiguities due to decimation are visible.

\section{CONCLUSION}

A data volume reduction technique is analysed, which is also applicable to the staggered SAR acquisition mode. The technique allows a significant reduction of the data volume for systems employing a PRF much larger than the processed bandwidth, with negligible degradation of the AASR. The technique is currently considered, together with the staggered SAR mode, for Tandem-L [5]. 